\title{
Operating in "Strange New Worlds" and Measuring Success - Test and Evaluation in Complex Environments
}

\author{
Garry Qualls ${ }^{1}$, Charles Cross ${ }^{2}$, Matthew Mahlin ${ }^{3}$, Gilbert Montague ${ }^{4}$, Mark Motter ${ }^{5}$, James Neilan ${ }^{6}$, Paul Rothhaar ${ }^{7}$, \\ Loc $\operatorname{Tran}^{8}$, Anna Trujillo ${ }^{9}$, and B. Danette Allen ${ }^{10}$ \\ NASA Langley Research Center, Hampton, Virginia 23681
}

\begin{abstract}
Software tools are being developed by the Autonomy Incubator at NASA's Langley Research Center that will provide an integrated and scalable capability to support research and non-research flight operations across several flight domains, including urban and mixed indoor-outdoor operations. These tools incorporate a full range of data products to support mission planning, approval, flight operations, and post-flight review. The system can support a number of different operational scenarios that can incorporate live and archived data streams for UAS operators, airspace regulators, and other important stakeholders. Example use cases are described that illustrate how the tools will benefit a variety of users in nominal and off-nominal operational scenarios. An overview is presented for the current state of the toolset, including a summary of current demonstrations that have been completed. Details of the final, fully operational capability are also presented, including the interfaces that will be supported to ensure compliance with existing and future airspace operations environments.
\end{abstract}

\section{Nomenclature}

$A E O N \quad$ Autonomous Entity Operations Network

AI Autonomy Incubator

$C 2 \quad$ Command and Control

CERTAIN City Environment for Range Testing of Autonomous Integrated Navigation

COA Certificate of Authorization

$D D S \quad$ Data Distribution Service

FAA Federal Aviation Administration

GCS Ground Control Station

GPS Global Positioning System

LaRC Langley Research Center

MOA Military Operations Area

$N A S \quad$ National Airspace System

UAS Unmanned Aircraft System

UAV $=$ Unmanned Aerial Vehicle

1 Senior Research Engineer, Aeronautics Systems Engineering Branch, AIAA Member.

2 Software Engineer, Crew Systems and Aviation Operations.

3 NASA Intern, Spring 2015, University of Nebraska-Lincoln, Lincoln, NE 68588.

4 NASA Intern, Spring 2015, Baldwin Wallace University, Berea, OH 44017.

5 Senior Research Engineer, Electronics Systems Branch, AIAA Member.

6 Computer Engineer, Flight Software Systems Branch.

${ }^{7}$ Research Engineer, Dynamic Systems and Controls Branch.

8 Computer Engineer, Flight Software Systems Branch.

9 Senior Research Engineer, Crew Systems and Aviation Operations, AIAA Member.

${ }^{10}$ NASA Langley Autonomy Incubator Lead, Crew Systems and Aviation Operations, AIAA Senior Member. 


\section{Introduction}

The NASA Langley Research Center (LaRC) Autonomy Incubator (AI) is developing a test and evaluation capability that encompasses both indoor and outdoor operating environments and employs vehicles with takeoff weights ranging from less than one ounce to more than 30 pounds $^{1}$. There are a few different flight test environments available for use at LaRC depending on the specific needs of a project and the technical and operational maturity of the Unmanned Aircraft System (UAS) being used.

\section{Flight Test Environments}

An indoor flight range with a netted operations volume that measures $84^{\prime}$ long, 42' wide, and $19.5^{\prime}$ high is available and includes an optical tracking system that can track multiple vehicles in real-time, providing their positions and orientations at rates above $200 \mathrm{~Hz}$. The AI also has a portable optical tracking system that can be easily relocated and configured to track vehicles in volumes as large is $30^{\prime} \times 30^{\prime} \times 8^{\prime}$ high. This allows research flights to be conducted in any other facilities that may be of interest, such as a wind tunnel test section or a stairwell.

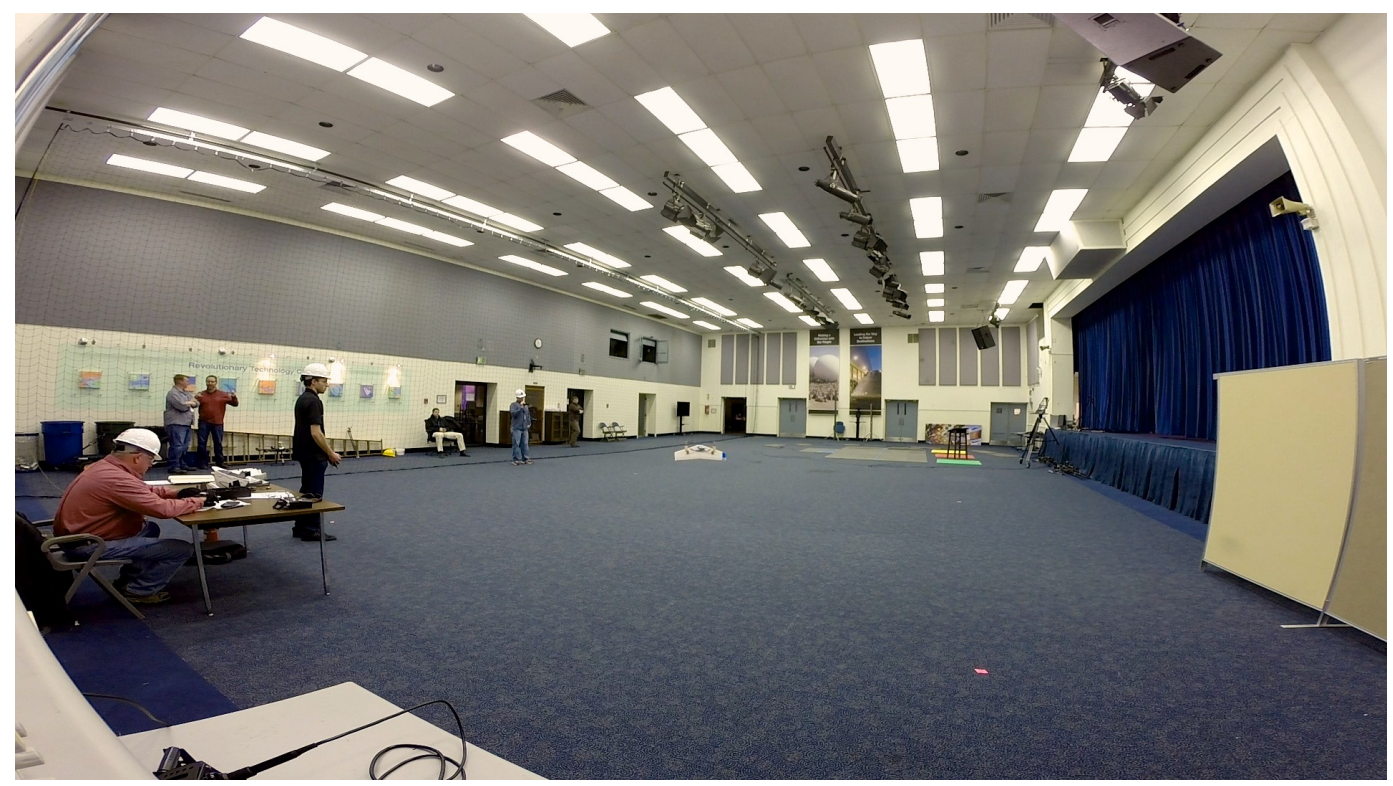

Figure 1. Indoor flight range of the NASA Langley Research Center Autonomy Incubator

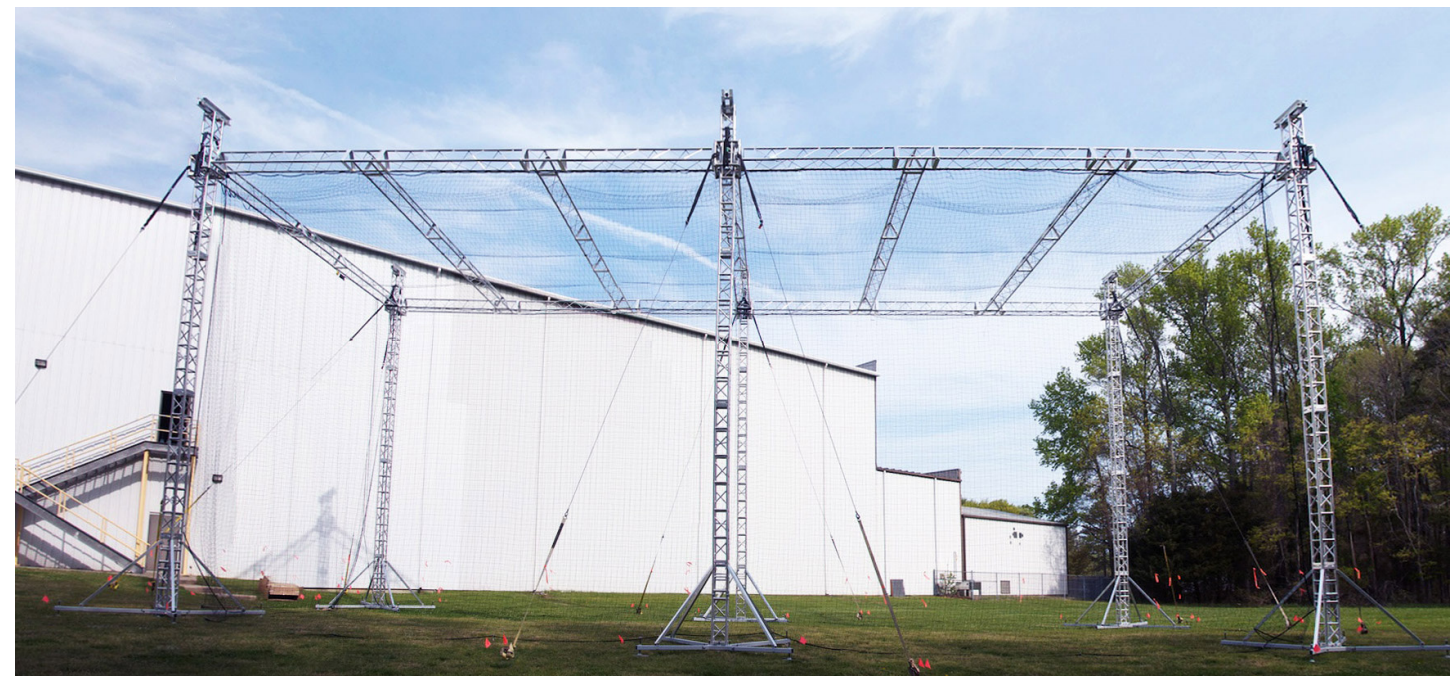

Figure 2. Outdoor, net-enclosed flight facility to support integration testing.

American Institute of Aeronautics and Astronautics 
To facilitate vehicle integration test flights that might be needed to safely transition a UAS from indoor flight testing to free flight outdoors in the National Airspace System (NAS), the AI has an outdoor facility that is fully enclosed by netting. This facility encloses a flight volume that measures $75^{\prime}$ long, $28^{\prime}$ wide, and $32^{\prime}$ high. Tethered outdoor flight is also used, on occasion, to support this kind of transitional integration testing.

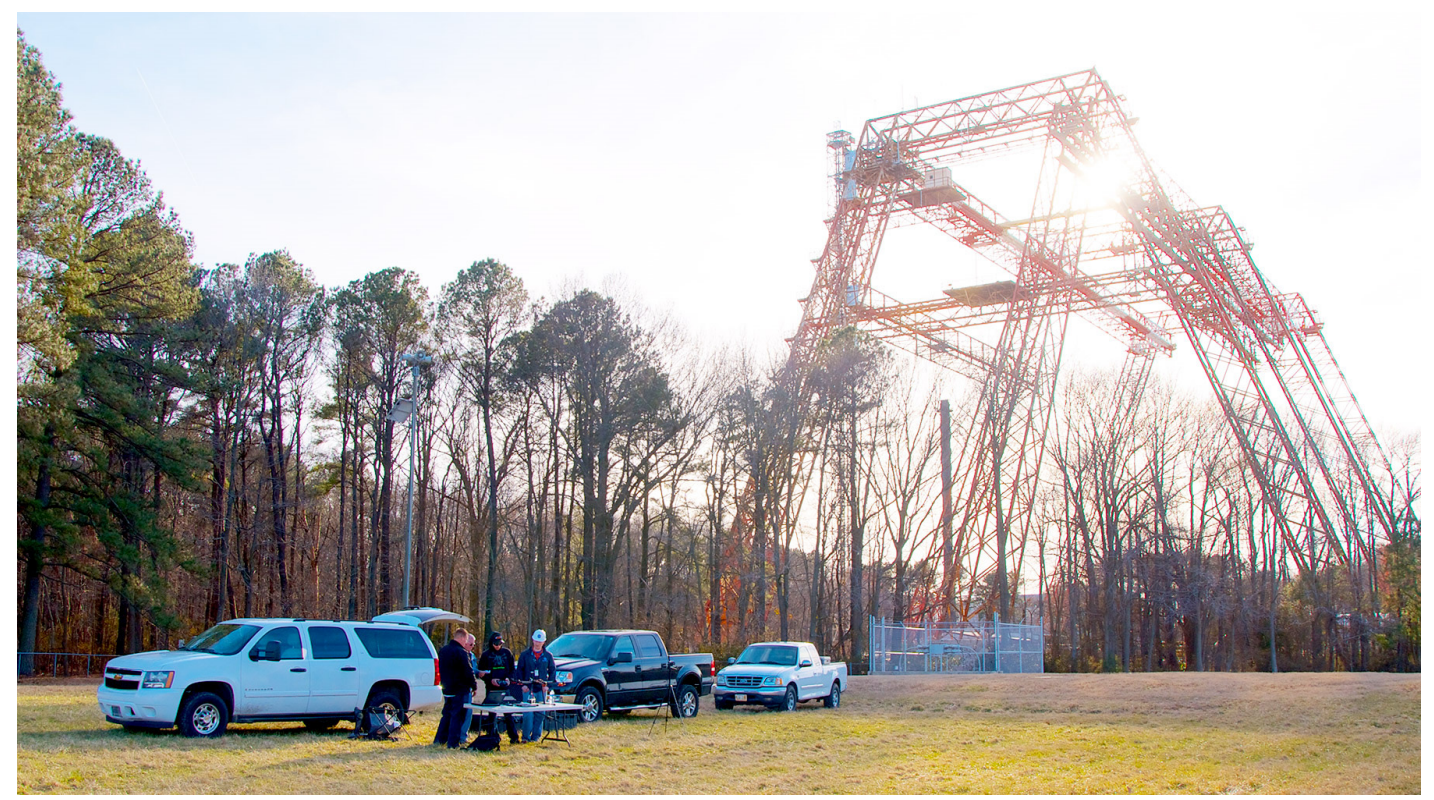

Figure 3. Preparations for a tethered hexacopter flight test in the CERTAIN flight range.

When appropriate, the AI can conduct free flights outdoors, in a variety of flight environments that are used regularly in support of LaRC UAS flight projects. The outdoor flight environments include restricted airspace within Military Operations Areas (MOAs), exercising agreements in place between NASA and the Department of Defense. NASA has an active Memorandum of Agreement with the Federal Aviation Administration (FAA) that authorizes UAS operations in Class $\mathrm{G}$ airspace, subject to the requirements specified in the memorandum. LaRC also has several active Certificates of Authorization (COAs) that have been issued by the FAA that provide for free flight of UAS at particular sites, subject to the specific requirements of each COA. This includes a COA that has been established as part of LaRC's CERTAIN (City Environment for Range Testing of Autonomous Integrated Navigation) activity and allows free flight of compliant UAS at altitudes up to 400' over parts of LaRC. To enable and support this work, the $\mathrm{AI}$ is developing and integrating the software tools and underlying frameworks that are needed to make research flights in these different domains safe and productive.

\section{Flight Test Operations}

Safety is a primary concern for any testing environment and monitoring the trajectories of all vehicles during testing is a fundamental requirement for safe operations and critical to effective test and evaluation activities. The basic components of an indoor multi-rotor test environment include a position estimation service that is used to monitor the vehicle performance and, optionally, inform the vehicle's control system of its position and orientation in real-time. The AI indoor environment includes a commercially-available optical tracking system that tracks reflective markers on each vehicle to produce an estimate of the vehicle's position and orientation in a locallydefined $(\mathrm{x}, \mathrm{y}, \mathrm{z})$ coordinate system. The tracking system provides position and orientation data for properly marked vehicles that are within visual range of the system's tracking cameras. 


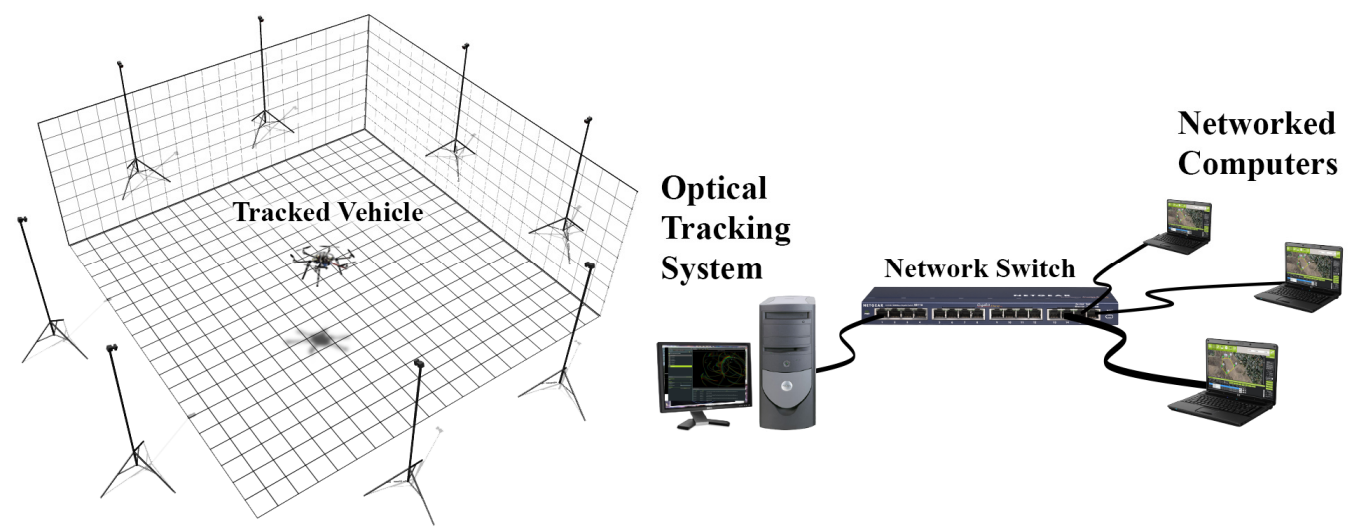

Figure 4. A portable optical tracking system broadcasts position and orientation estimates for all tracked vehicles to any networked computers that subscribe to its data stream.

The raw position and orientation data for vehicles visible to the optical tracking system are published over a computer network and available to any network-connected devices that have subscribed to that data stream. A realtime estimate of the latency of the each position and orientation estimate, along with the precise time that each measurement was received, are also published over the network. Except in special cases, real-time communication of tracker system data to computers or other subsystems onboard the vehicles must be done wirelessly.

Similarly, for outdoor operations, a vehicle position estimation service is required to enable the tracking of all vehicles in real-time. The most commonly used outdoor position estimation service is the Global Positioning System (GPS), which enables a compatible GPS receiver to estimate its position in a global coordinate frame. As with the indoor position estimation service, the GPS-based position estimates have a latency associated with each measurement but, unlike the indoor system, most GPS receivers do not estimate their latency in real-time or make latency estimates available to users. GPS receiver output does, however, include data to indicate the quality of the current solution.

Since a vehicle's GPS position estimate is generated onboard, it is usually transmitted wirelessly to any vehicles or operators that need to establish and maintain situational awareness of the vehicle's location. It is very common for outdoor flight vehicles to incorporate an autopilot that includes the GPS-based position estimates in its standard Command and Control (C2) telemetry data stream. This allows the autopilot's Ground Control Station (GCS) to display the location of the vehicle to its operators in near real-time and allows the vehicle's trajectory, along with all other telemetered data, to be logged for post-flight analysis. For outdoor flight vehicles that do not include an autopilot, a separate GPS tracking and telemetry system can be added to the vehicle to enable vehicle position tracking to the accuracy of the GPS receiver's solution.

The optical tracking and GPS tracking systems described above are the most common methods used to track research flight vehicles in their respective environments and the underlying operating principles of each have very little in common. They are, however, quite similar, functionally, in that each of these tracking systems provides a series of position estimates for any properly equipped vehicle. Additionally, when working properly, each provides estimates with sufficient update rate, accuracy, and latency to have become the basis of several standard vehicle management processes that are critical for the planning and execution of safe flight operations as well as the acquisition of high-quality test data. 
In non-ideal cases, as the quality of either system's position estimates begin to degrade, the safety of flight operations and the quality of test data can degrade to unacceptable levels. This case can be extended further to nonresearch applications, such as commercial UAS operations in complex urban environments or in mixed indooroutdoor operations, where the quality of GPS-based navigation solutions are known to degrade significantly. This highlights the importance of developing a UAS operations approach that can maintain acceptable levels of safety when position estimate quality drops below acceptable levels or the ability to estimate a vehicle's position is lost altogether. This alignment of the needs to a) maintain safe operations across all of the flight environments being

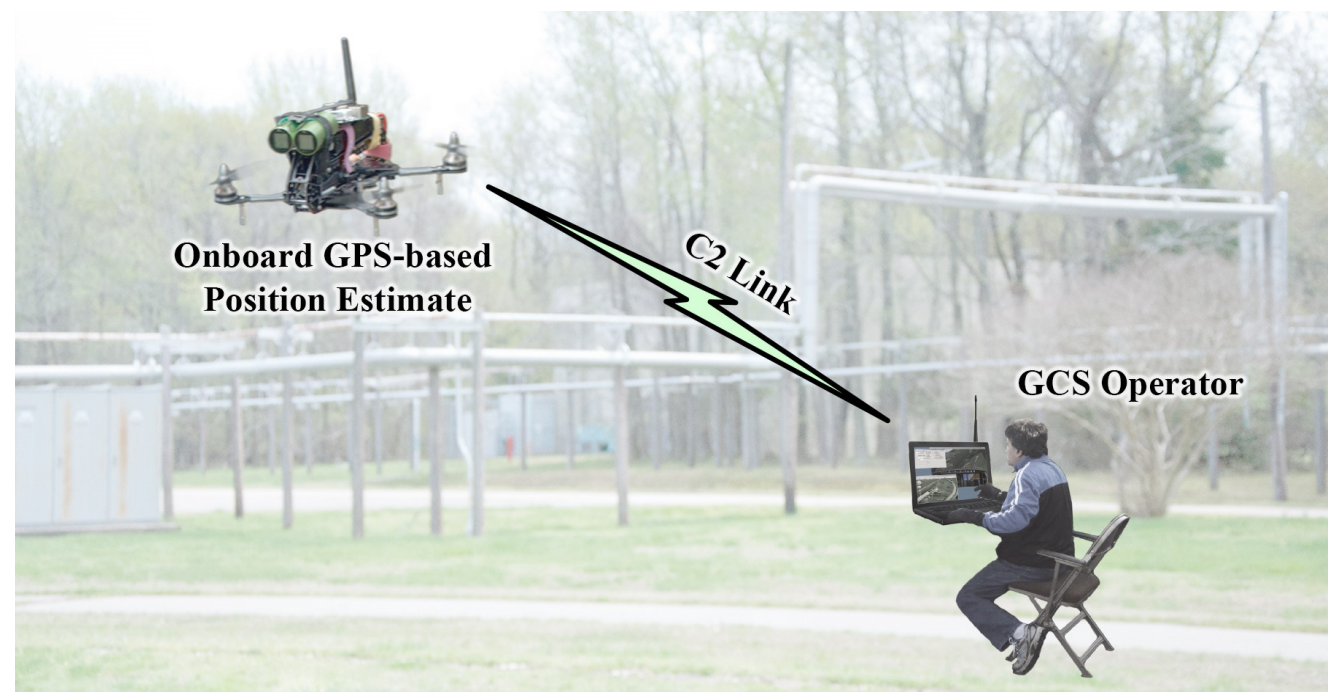

Figure 5. UAVs typically generate their own position estimates using onboard GPS receivers when operated outdoors.

used by the AI, b) determine when system performance has dropped below levels needed to produce acceptable test data, and c) field systems that can operate safely and reliably in urban environments, further emphasizes the importance and value of developing an extensible, standards-compliant UAS flight management framework. To be a complete solution, the framework must be integrated with a complimentary concept of UAS operations to produce safe operations across a broad range of current research and future commercial scenarios.

\section{Flight Test Management}

The AI is developing an extensible and standards-compliant UAS flight management framework, named the Autonomous Entity Operations Network $(\mathrm{AEON})^{2}$, to support a broad array of research activities that span indoor and outdoor operational environments, employ ground and air vehicles, and can easily integrate commercially available vehicle systems and more exotic systems, whether they have been developed in-house or elsewhere. The framework consists of well-defined interfaces, standards-compliant data transport mechanisms, and system management tools that communicate heterogeneous wired and wireless networks. The modular components of the framework are deployable in forms that are appropriate for computers ranging from tiny, onboard microcontrollers to large, cloud-based clusters. Demonstrations have shown that the communication services implemented provide enough speed and bandwidth to support onboard, inner-loop vehicle control research ${ }^{3}$. 


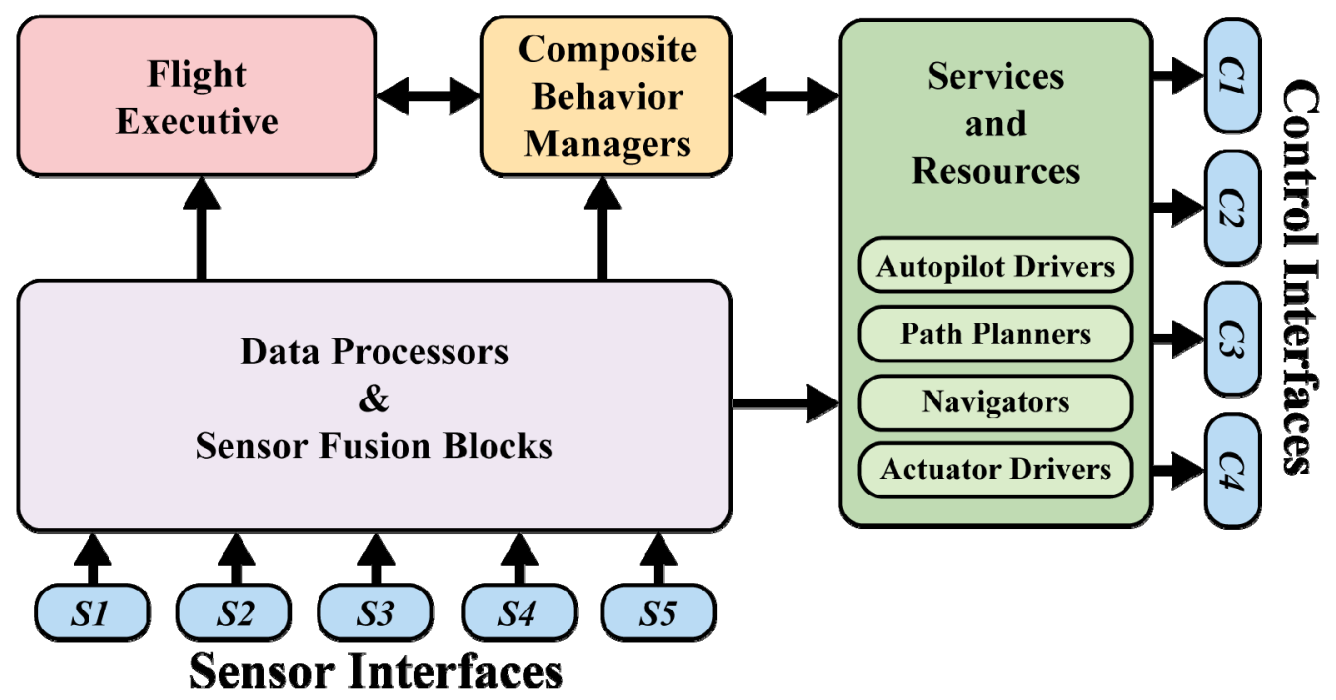

Figure 6. A block diagram of the AEON Architecture.

Work to date has focused on the safe management of indoor flights and enabling research on a diverse range of topics that include machine learning ${ }^{4,5,6}$, machine vision systems, automated route planning, and inner loop control. Prominent features of the current framework include use of the Data Distribution Service (DDS) as the data distribution layer of the framework and implementing bridges between DDS and other popular robotics frameworks and data transmission protocols.

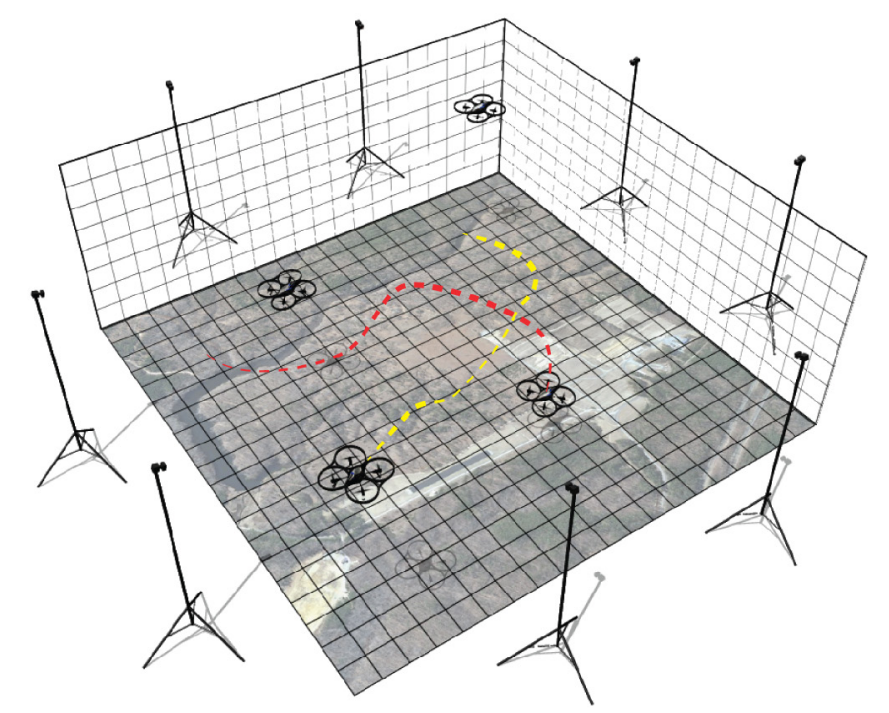

Figure 7. The physics-based simulation environment integrates live and simulated data to support the development of automated route planning algorithms and assist with machine vision research.

A simulation environment has been implemented as part of the automated route planning research and it has been integrated with the tools used for real-time flight operations management. The modularity of the framework components and the high performance data transport services provide much needed flexibility for research efforts that need to advance quickly. Early examples demonstrated the ability to merge elements from a simulated 
environment with elements of an actual flight demonstration in real-time. This allowed the researcher to easily create scenarios in which a real helicopter in the indoor flight range could navigate to avoid virtual obstacles imported from the simulation or have a simulated helicopter navigate relative to real obstacles that were being tracked in the indoor range.

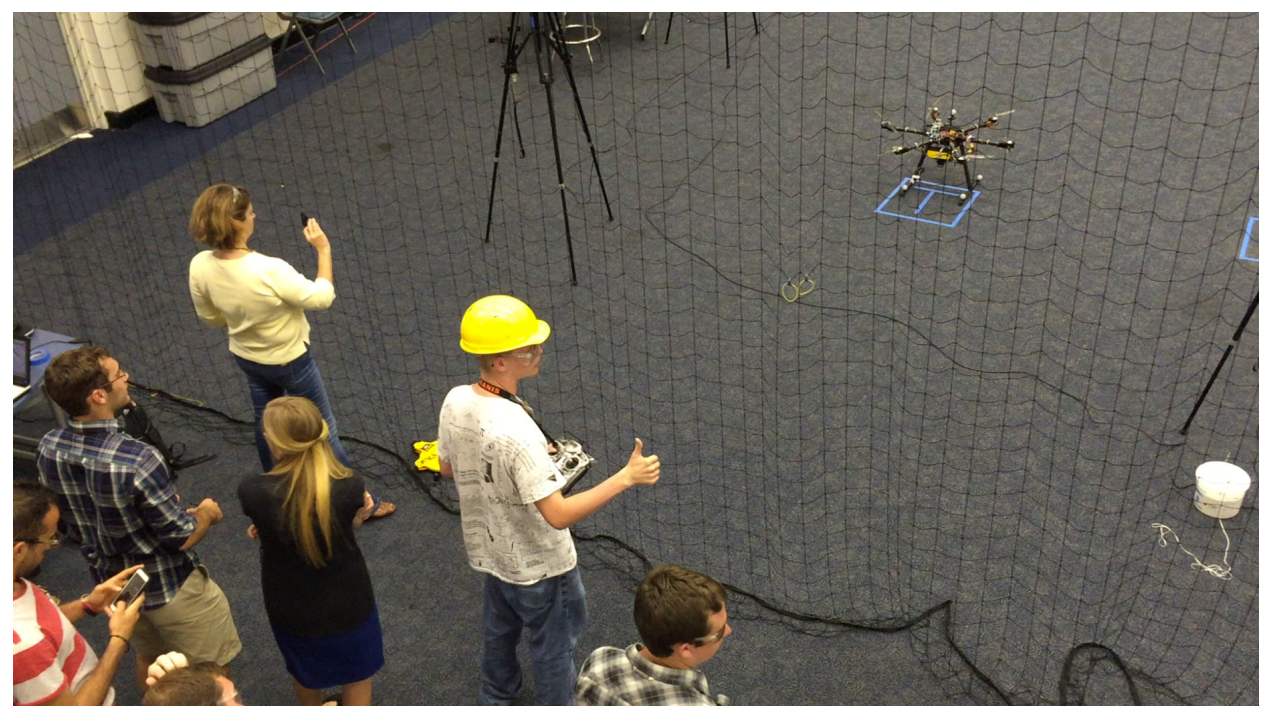

Figure 8. A recent GPS Emulator demonstration in which a $12 \mathrm{lb}$. hexacopter that was configured for outdoor, GPS-assisted flight, was able to navigate successfully indoors with no GPS signal and no changes to its onboard software.

A current AI focus is the emulation of GPS receiver performance in the AI's indoor flight range. This enables stress testing of the vehicle autopilot and ground control station to evaluate the system's response to specific GPS failures and errors. Doing this kind of testing in a well-controlled, indoor environment will allow researchers to greatly improve test repeatability as well as measure test results with a much higher degree of accuracy than could be achieved outdoors.

\section{Future Work}

Future work will encompass a number of topics related to safe operations and the ability to conduct flight research efficiently ${ }^{7}$. The GPS Emulator will be expanded to emulate other popular GPS receivers and expanded to other types of vehicles. There are several changes to lab concepts of operation that will be evaluated in concert with the expanding capabilities of the AEON framework. These include digital checklists that are executed prior to takeoff and system health checks that are performed just after takeoff. Also being considered are the implementation of highly diverse, redundant communication links, including Internet connectivity and the use of cloud services. Compatibility with the FAA's Cloud Computing Strategy would be evaluated, along with evaluation of the potential impacts on the system that could result from cyber attacks. Expanding the use of software testing to provide higher reliability is also being pursued.

\section{Conclusion}

The current capabilities of the AI have been planned and implemented using technologies that provide flexibility for both researchers and software developers. The safety and data quality challenges that must be met for the AI to succeed mirror many of the challenges that must be addressed to enable routine commercial UAS operations in urban and mixed indoor-outdoor environments. The solutions being developed within the AI are being developed and evaluated with an eye toward these problems that must be solved before UAS operations in complex environments become safe and routine. 


\section{References}

${ }^{1}$ Allen, D., Cross, C., Tran, L Motter, M., Neilan, J., Qualls, G., Rothhaar, P., Trujillo, “Towards Safe Robust Autonomous Operations", AIAA 2015 Special Session Invited Paper.

${ }^{2}$ Cross, C., Motter, M., Neilan, J., Tran, L Qualls, G., Rothhaar, P., Trujillo, A., Allen, D., "It's Difficult to work in groups when you're Omnipotent, So don't be - An Open, Distributed Software Architecture for UXS Operations”, AIAA 2015 Special Session Invited Paper.

${ }^{3}$ Rothhaar, P., Cross, C., Tran, L Motter, M., Neilan, J., Qualls, G., Trujillo, A., Allen, D., “A Flexible Flight Control System for Rapid GNC and Distributed Control Development", AIAA 2015 Special Session Invited Paper.

${ }^{4}$ Tran, L., Cross, C., Motter, M., Neilan, J., Qualls, G., Rothhaar, P., Trujillo, A., Allen, D., "Reinforcement Learning with Autonomous Small Aerial Vehicles in Cluttered Environments", AIAA 2015 Special Session Invited Paper.

${ }^{5}$ Neilan, J. H., Cross, C. D., Motter, M. A., Qualls, G. D., Rothhaar, P. M., Trujillo, A. C., and Allen, B. D., "Using Multimodal Input for Autonomous Decision Making", AIAA 2015 Special Session Invited Paper.

${ }^{6}$ Motter, M., Neilan, J., Cross, C., Tran, L., Qualls, G., Rothhaar, P., Trujillo, A., Allen, D., "His Pattern Indicates TwoDimensional Thinking - Deciding to Go Around via Machine Learning", AIAA 2015 Special Session Invited Paper.

${ }^{7}$ Trujillo, A., Cross, C., Tran, L Motter, M., Neilan, J., Qualls, G., Rothhaar, P., Allen, D., “I'm a Doctor, Jim Not an Engineer - Collaborating with Autonomous Agents", AIAA 2015 Special Session Invited Paper. 